\title{
Bi-LSTM Sentiment Classifier for Climate Change Issues in South Korea
}

\author{
Do-Yeon Kim, Sung-Won Kang, Seong-Taek Park
}

\begin{abstract}
A sentiment analysis using SNS data can confirm various people's thoughts. Thus an analysis using SNS can predict social problems and more accurately identify the complex causes of the problem. In addition, big data technology can identify SNS information that is generated in real time, allowing a wide range of people's opinions to be understood without losing time. It can supplement traditional opinion surveys. The incumbent government mainly uses SNS to promote its policies. However, measures are needed to actively reflect SNS in the process of carrying out the policy. Therefore this paper developed a sentiment classifier that can identify public feelings on SNS about climate change. To that end, based on a dictionary formulated on the theme of climate change, we collected climate change SNS data for learning and tagged seven sentiments. Using training data, the sentiment classifier models were developed using machine learning models. The analysis showed that the Bi-LSTM model had the best performance than shallow models. It showed the highest accuracy (85.10\%) in the seven sentiments classified, outperforming traditional machine learning (Naive Bayes and SVM) by approximately $34.53 \%$, and $7.14 \%$ respectively. These findings substantiate the applicability of the proposed Bi-LSTM-based sentiment classifier to the analysis of sentiments relevant to diverse climate change issues.
\end{abstract}

Keywords : Climate Change, Machine Learning, Bi-LSTM, CNN, Sentiment Classifier

\section{INTRODUCTION}

In a bid to counteract the natural disasters linked to climate change, the government reports real-time weather conditions and implements other responsive policy measures. Yet in the process, it is prone to encounter some challenges resulting from the lack of public trust [1]. To address such challenges, it is necessary to develop public engagement policies and to give prompt feedback to public opinions [2]. Real-time accessibility to the information of SNS by means of big data analytics could enable government authorities to grasp diverse public opinions. SNS-based analyses reveal thoughts of different stakeholders, increasing the accuracy in predicting social issues and illuminating the complex causality thereof [3]. The government needs to

Revised Manuscript Received on July 22, 2019.

Do-Yeon Kim, Researcher,Korea Environment Institute, 370 , Sicheong-daero, Sejong-si, 30147, Republic of Korea. Email: dykim@kei.re.kr

Sung-Won Kang*, Senior Research Fellow, Korea Environment Institute, 370, Sicheong-daero, Sejong-si, 30147, Republic of Korea. Email: swkang@kei.re.kr

Seong-Taek Park, Professor, Sungkyunkwan University, 25-2, Seonggyungwan-ro, Seoul, 03063, Republic of Korea. Email: solpherd@skku.edu

actively reflect SNS in the entire process of policy implementation. In that regard, this paper proposes a sentiment classifier focused on the public perception of climate change issues on SNS(Facebook, Twitter and Instagram) and in news comments.

\section{RELATED WORKS}

With the needs for data analysis and problem-solving diversifying, the classification models based on machine learning extend to non-linear models from linear ones. Also with the advancement of GPU computing and massive data collection and storage technologies, neural network models are drawing much attention as a way of increasing the complexity of models with ease. CNN (Convolutional Neural Network) and RNN (Recurrent Neural Network) are most widely used neural networks [6]. In natural language processing, CNN uses 1D convolution and understands the spatial correlation comparable to a bag of n-grams [9]. RNN can extract the temporal correlation inherent in texts [4]. Recently, the methodologies for classification of sensibility based on machine learning are being studied, and they show excellent classification performance [Table 1].

Table- I: Sentiment Classification Study Based on Machine Learning

\begin{tabular}{|c|c|c|}
\hline Literature & $\begin{array}{c}\text { Methods / } \\
\text { Data / } \\
\text { Sentiment class } \\
\end{array}$ & Description \\
\hline \multirow{3}{*}{$\begin{array}{l}\text { Pang et al. } \\
\text { (2002) }\end{array}$} & $\begin{array}{l}\text { Naïve Bayes, SVM } \\
\text { Maximum Entropy, }\end{array}$ & \multirow{3}{*}{$\begin{array}{l}\text { - Comparing classification through various } \\
\text { combinations, Model with } 1 \text { syllable and } \\
\text { SVM combined was the highest performing } \\
\text { with } 82.9 \% \text { accuracy [5] }\end{array}$} \\
\hline & Movie Review & \\
\hline & Positive, Negative & \\
\hline \multirow{3}{*}{$\begin{array}{l}\text { Wang et al. } \\
\text { (2016) }\end{array}$} & $\begin{array}{l}\text { LSTM, AE-LSTM, } \\
\text { AT-LSTM, } \\
\text { ATAE-LSTM } \\
\end{array}$ & \multirow{3}{*}{$\begin{array}{l}\text { - ATAE-LSTM model shows the highest } \\
\text { classification accuracy of } 84.0 \% \text { in } 3 \\
\text { categories and } 89.9 \% \text { in } 2 \text { categories [7] }\end{array}$} \\
\hline & Customer Review & \\
\hline & $\begin{array}{l}\text { Positive,Negative,Neutra } \\
\text { lity }\end{array}$ & \\
\hline \multirow{3}{*}{$\begin{array}{l}\text { Cliché } \\
\text { (2017) }\end{array}$} & $\begin{array}{c}\text { CNN, LSTM, } \\
\text { CNN and LSTM } \\
\end{array}$ & \multirow{3}{*}{$\begin{array}{l}\text { - Ensemble models combined with CNN and } \\
\text { LSTM were the highest performing models } \\
\text { with an accuracy of } 74.8 \%[8]\end{array}$} \\
\hline & Twitter & \\
\hline & Positive, Negative & \\
\hline \multirow{3}{*}{$\begin{array}{l}\text { Sosa } \\
(2017)\end{array}$} & $\begin{array}{l}\text { CNN, LSTM, CNN and } \\
\text { LSTM, LSTM and CNN }\end{array}$ & \multirow{3}{*}{$\begin{array}{l}\text { - Ensemble models combined with } \\
\text { LSTM-CNN were the highest performing } \\
\text { models with accuracy of } 75.2 \%[10]\end{array}$} \\
\hline & Twitter & \\
\hline & Positive, Negative & \\
\hline
\end{tabular}




\section{Bi-LSTM Sentiment Classifier for Climate Change Issues in South Korea}

The classification performance varies with the features of data. Thus, given the diversity of subjects whose sentiments were analyzed and the theme 'Climate Change' being short-sentence data in Korean, no particular machine learning wasfound be suitable in this study. Therefore, utilizing the shallowmodels (Naive Bayes and SVM) and the emerging deep learning (CNN and Bi-LSTM), this study developed a sentiment classifier, which fits the features of the subjects analyzed. Also, instead of a simple Binary classification, positivity is classified into three categories (ecstasy, expectation, and admiration). Also, negativity is classified into 3 categories (rage, terror and sad). The absence of sentiments is classified as neutrality. In short, a total of seven sentiments were classified in this study.

\section{PROPOSED METHOD}

The scope and flow diagram of this study are as follows in Figure 1. First, a dictionary was established on the topic of 'Climate Change'. The process of building a climate change dictionary is a necessary step for collecting training data set for building sentiment classifiers. The dictionary was established by classifying natural disasters due to climate change into four categories: temperature, precipitation, land and oceans. Based on the dictionaries built in this study, we collected about 50,000 posts and short stories from Facebook, Twitter, Instagram and Naver News Comments. These social data have a limited number of characters to write, so sentences are short and difficult to understand. SNS data also uses many new words, connotations, slangs, and acronyms, as well as writing and spelling errors. Recently, it has been using emoji a lot instead of letters. Therefore, it is difficult to digitize SNS data for machine learning. Therefore, this study performed analyses that matched the characteristics of these social data. First, seven sentiments (ecstasy, expectation, admiration, rage, terror, sad and neutrality) were tagged on the subjective judgment of a person to establish training data. Based on the training data set deployed, pre-processing such as corpus generation, normalization, and emoji conversion to Korean were performed and finally each SNS data was reviewed case by case to fix anomalies. Based on this training data set, we applied support vector machine (SVM) and Naive Bayes to build sentiment classifier algorithms. In addition, a classification algorithm was developed using CNN (Convolutional Neural Networks) and Bi-LSTM (Bidirectional LSTM) among the deep learning techniques. Finally, we selected the model with highest accuracy by comparing the classification performance.

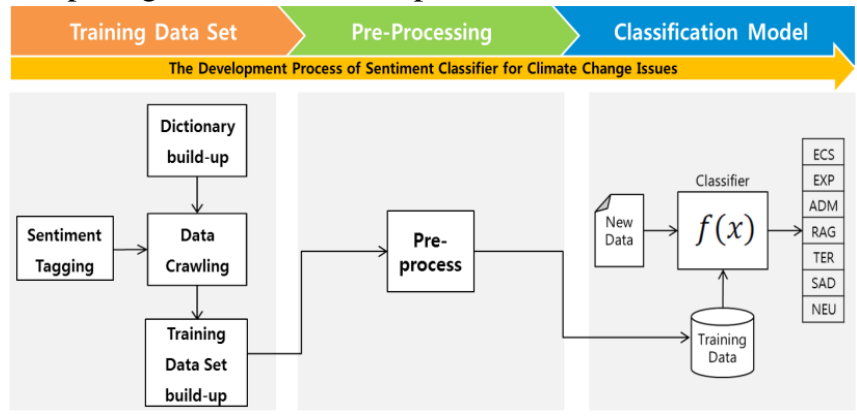

Fig 1. Analysis Process

\section{A. CNN Model}

The CNN model processes the words $\left\{w_{1_{\operatorname{man}}} w_{t}\right\}$ that constitute the nth sentence, $S_{n}$ through embedding layers. The $\mathrm{CNN}$ is defined as in formula (1).

$$
\begin{gathered}
x_{1-t}=x_{1} \oplus x_{2} \oplus \cdots \oplus x_{t} \\
c_{i}=f\left(w \cdot x_{1-1+h-1}+b\right) \\
c=\left[c_{1}, c_{2},{ }^{\cdots x}, c_{t-\hat{h}+1}\right] \\
\hat{c}=\max \{c\}
\end{gathered}
$$

In the above formula (1), $\oplus$ is the Concatenation that connects the vectors. $w$ means the filter included in the Convolutional operator. $h$ means window size and is a parameter that determines how many words are used at a time within a sentence when extracting features. A total of $t-h+1$ features are extracted in one sentence. After the max pooling process, $\hat{\epsilon}$ will be printed out as a feature for the sentence. Features extracted from these processes are used for sentiment classification. Figure 2 below shows the structure of the CNN classifier. First sentence entered and converted to a vector in word units through the embedded layer. Converted vectors are entered into the Convolutional layer to allow the model to learn. In the Convolution layer, features that represent the text entered through the Convolution operation are extracted.These extracted features leave only important information through the max pooling layer, and the final features are printed out as inputs to the next layer.There are many different methods for pooling, such as Mean Pooling, Average Pooling and Max Pooling.In classification models, Max Pooling is typically used to extract the largest of the features extracted through the Convolution.

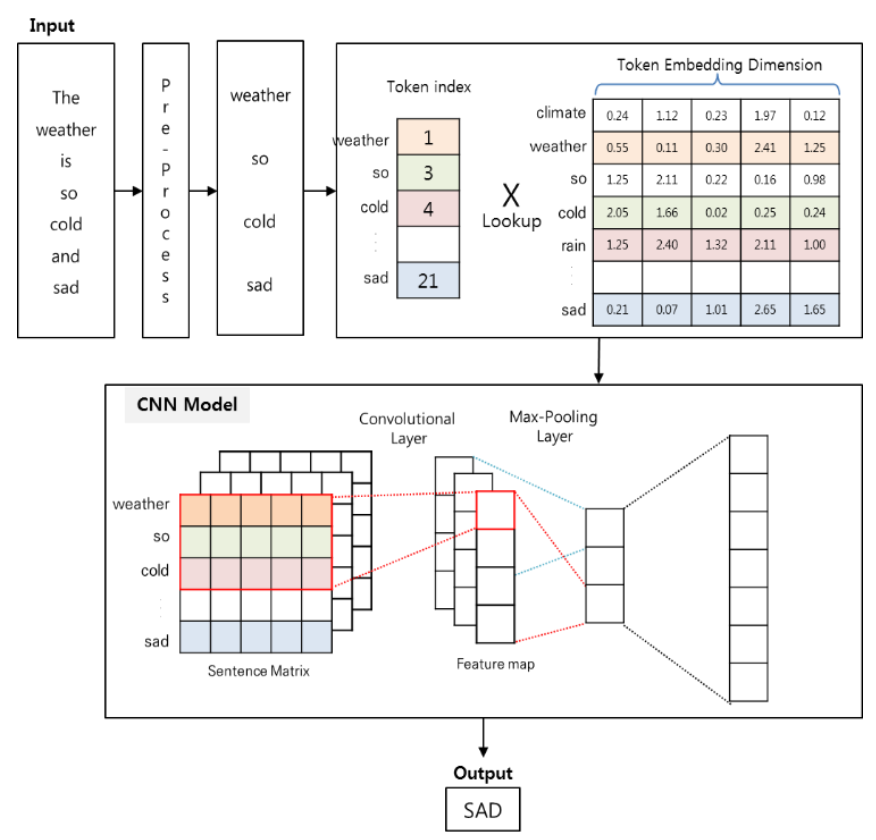

Fig 2. CNN Classifier Model

The inputs of the $\mathrm{CNN}$ model developed in this study include 
diverse parameters, e.g. epoch, sentence, filter and kernel. The algorithm used three filters of size tree, four and five filter with ReLU used for activation. Also, 30\% drop-out is used to minimize the over-fitting issues of the classification model [figure 3].

CNN Classifier(

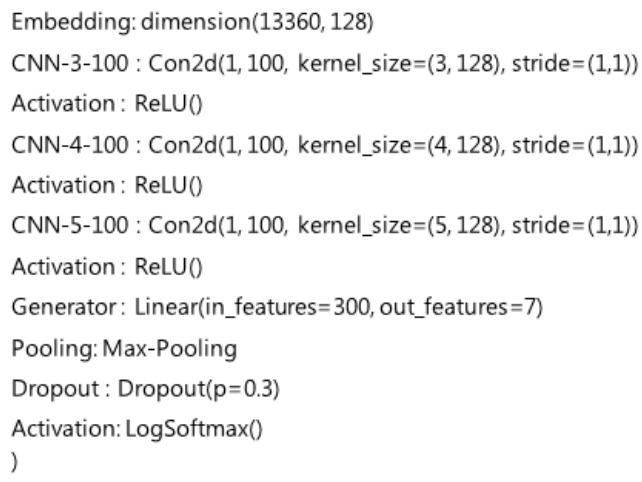

Fig 3. CNN Classifier Training Algorithm

\section{B. Bi-LSTM Model}

The LSTM (Long Short Term Memory) addresses the challenges of RNN's gradient vanishing, and shows great performance in language and voice recognition as reported in the literature aforementioned. LSTM is defined as in formula (2).

$$
\begin{gathered}
\left.i_{t}=\sigma\left(W_{t} x_{t}+W_{h i} h_{t-1}+W_{c i} c_{t-1}+b_{i}\right)\right) \\
f_{t}=\sigma\left(W_{x f} x_{t}+W_{h f} h_{t-1}+W_{c f} c_{t-1}+b_{f}\right) \\
c_{t}=f_{t} C_{t-1}+i_{t} \tanh \left(W_{x e} x_{t}+W_{h c} h_{t-1}+b_{c}\right) \\
o_{t}=\sigma\left(W_{x 0} x_{t}+W_{h o} h_{t-1}+W_{c o} c_{t-1}+b_{0}\right) \\
h_{t}=o_{t} \tanh \left(c_{t}\right) \\
y_{t}=g\left(W_{x y} h(t)+b_{y}\right)
\end{gathered}
$$

In above formula (2), $\sigma$ is the Sigmoid function, and $i, f$, $o$ and $c$ respectively represent input gate, memory cell vector and output gate.The size of each vector is equal to the size of the hidden layer vector.The Subscript of the weighting matrix means each node connected. $W_{h i}$ is a weighted matrix between the hidden layer( $h)$ and the input gate( $(i)$. Figure 4 below shows the structure of LSTM memory cell.

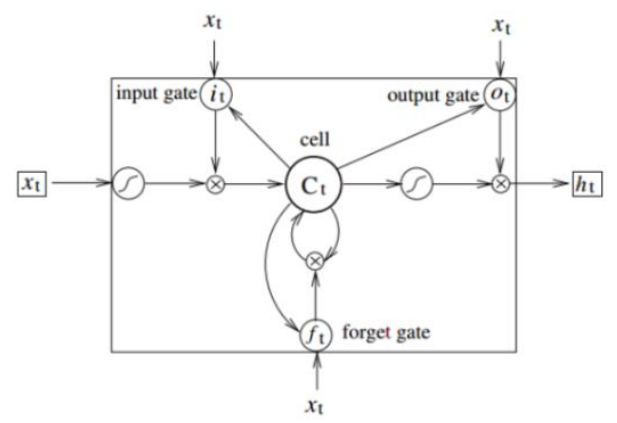

Fig 4. LSTM memory cell

These gates can adjust the values of newly entered vectors and existing cell vectors to maintain previously stored information, so there is no problem with the loss of gradient.However, the gate has the disadvantage of not knowing the information that appears after the current point in time.Korean has a structure in which descriptive words appear at the back of a sentence.Therefore, it may be difficult to detect descriptive information, which is important in determining semantics.In previous studies, the backward method was proposed based on the fact that the descriptor appears behind the character, showing higher performance than the existing LSTM model.However, this method has the disadvantage of not being able to see information throughout a sentence. Therefore, this study proposed Bidirectional LSTM to be able to identify both previous and next input words by learning LSTM in both directions to understand the entire sentence.

The following [Figure 5] shows the structure of the Bi-LSTM classification model built in this study. As with the CNN model discussed earlier, the entered sentence is converted to a vector in word units through the embedded layer.Translated vectors are entered sequentially into the Hidden layer of the Forward-LSTM to allow the model to learn.In the Hidden layer, both previous and current input values are considered to determine the status value of each step.Also, the previous input value is a structure that is reflected in the learning of the current input.Likewise, Backward layer was performed to learn training data set in both directions.LSTM solves the Gradient vanishing problem in RNN, allowing it to learn the Long-term dependency more effectively.

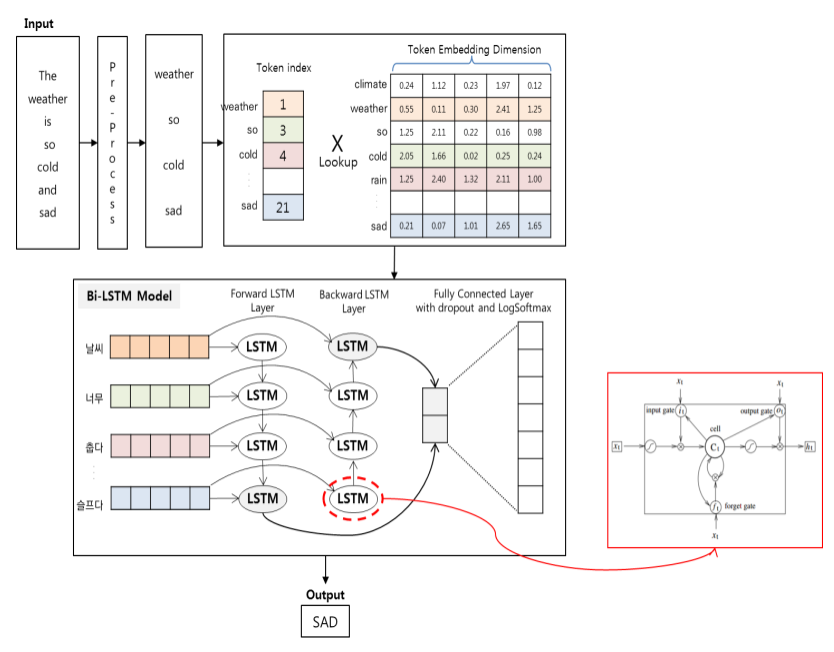

Fig 5. Bi-LSTM Classifier Model

The following [Figure 6] is a learning algorithm for the Bidirectional LSTM model. The input of the algorithm has various parameters such as Epoch, Sentence, and Hidden Layer. Learning algorithms are divided into forward pass and back pass. In addition, 30\% Drop-out was used to minimize the overfitting problem of classification models. 
Bi-LSTM Classifier(

Embedding: dimension $(13360,128)$

RNN: LSTM(num_layers $=4$, bidirectional $=$ True)

Generator : Linear(in_features $=512$, out_features $=7$ )

Dropout : Dropout $(\mathrm{p}=0.3)$

Activation: LogSoftmax()

)

\section{Fig 6. Bdirectional LSTM Classifier Training Algorithm}

\section{EXPERIMENTAL RESULTS}

The analysis showed that the highest accuracy of the Bi-LSTM model in 7 sentiments $(85.10 \%)$ and 3 sentiments (92.95\%) classified here. In contrast shallow models had low accuracy, for example Naïve Bayesian was least accurate in 7 sentiments (50.57\%) and 3 sentiments (65.73\%).

Table-II: Accuracy by sentiment classification

\begin{tabular}{|c|c|c|c|}
\hline \multicolumn{2}{|c|}{ Model } & $\mathbf{7}$ class & 3 class \\
\hline \multicolumn{2}{|c|}{ Naive Bayesian } & $50.57 \%$ & $65.73 \%$ \\
\hline \multirow{3}{*}{ SVM } & Linear kernel SVM & $77.96 \%$ & $87.25 \%$ \\
\cline { 2 - 4 } & RBF kernel SVM & $68.71 \%$ & $76.25 \%$ \\
\cline { 2 - 4 } & $\begin{array}{c}\text { Sigmoid kernel } \\
\text { SVM }\end{array}$ & $76.85 \%$ & $86.31 \%$ \\
\cline { 2 - 4 } & $\begin{array}{c}\text { Polynomial kernel } \\
\text { SVM }\end{array}$ & $75.04 \%$ & $84.08 \%$ \\
\hline \multirow{2}{*}{ CNN } & $81.95 \%$ & $90.65 \%$ \\
\hline \multicolumn{2}{|c|}{ Bi-LSTM } & $\mathbf{8 5 . 1 0 \%}$ & $\mathbf{9 2 . 9 5 \%}$ \\
\hline
\end{tabular}

Naive Bayesian has limitations in that it emphasizes whether simple words are present or not and ignores the word order. By contrast, the proposed Bi-LSTM converts the word-word relation information into a vector in the word embedding layer, and has synonyms placed at adjacent coordinates in higher dimensional space through learning. Also, as the overall contextual flow was learned bi-directionally in the order of words in the Bi-LSTM layers, the Bi-LSTM model using embedding showed the highest performance in this study.

The accuracy of the seven sentiment class of Bi-LSTM is as shown in Figure 7. The analysis showed that the non-sensitive neutrality had the highest classification accuracy of $89.8 \%$, as the positive emotion was classified into three categories: ecstasy, expectation, admiration and negative emotion were classified into three categories: Rage, Terror and sad. Ecstasy includes most common positive keywords such as good, smiling, happy, and pleasant. So it appears to have shown relatively low degree of classification accuracy. Likewise, Rage appears to have shown relatively low degree of classification accuracy, as it includes most common negative keywords such as hate, irritation, madness, and death.

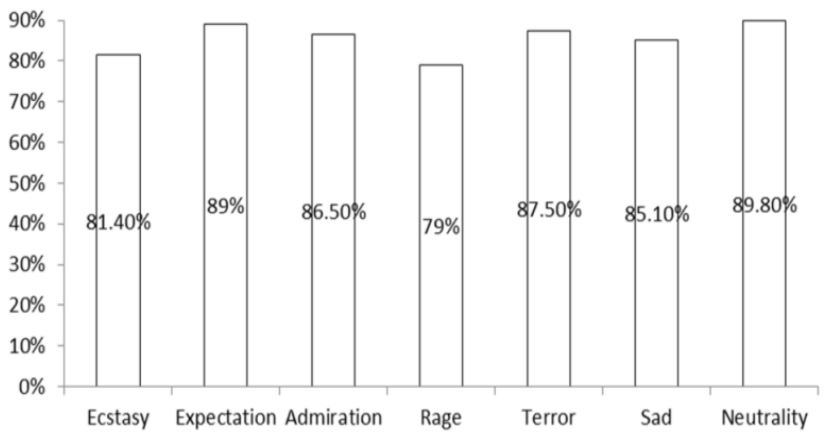

Fig 7. Accuracy of Classification by Sentimnet Class using Bi-LSTM

In addition, accuracy was analyzed by media for the Bi-LSTM with the highest classification accuracy. Training data set built in this study consists of four media: Facebook, Twitter, Instagram and News comments. The accuracy of each of the four media is shown in Figure 8.The analysis showed that the accuracy of classification was followed by Twitter (88.5\%), News Comments (87.3\%), Facebook $(85.6 \%)$, and Instagram (79.5\%). The classification accuracy by media is not significant, but the Instagram showed the lowest classification accuracy at $79.5 \%$.Since Instagram has more serious problem than other media about new words, slang, connotation, and floating language problems, it is believed to have shown lower classification accuracy.In addition, the rest of the media, except for Instagram, showed higher accuracy than the overall classification accuracy $(85.1 \%)$.Therefore, in the future, the classification accuracy will be higher if the sentiment classifier built with the media-specific training data set is used instead of the overall training data set.

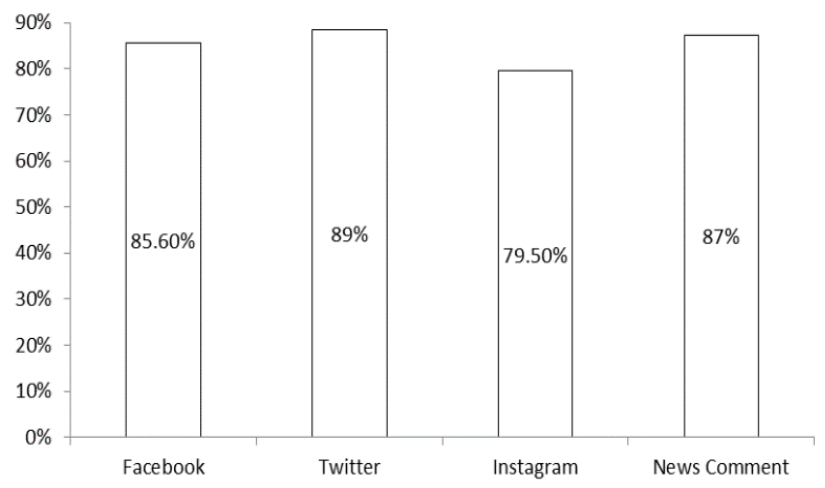

Fig 8. Accuracy of Classification by Media using Bi-LSTM

\section{CONCLUSION}

In this study, the sentiment analysis channels vary and the subject is phenomenon due to climate change. Therefore, it is not possible to define in advance that a particular machine learning algorithm is suitable for this study through prior study. Therefore, sentiment classification model for this study was developed using shallow models (SVM, Naïve Bayes) and deep learning techniques (CNN, Bidirectional LSTM) that performed well in recent natural language processing studies. 
To enhance the performance of the sentiment classification, the problem of lack of training data set was mitigated by converting text data into vectors through embedding. And Drop-out was used to minimize the problem of overfitting. The results of this study, Bi-LSTM outperformed the other models. It showed the highest accuracy $(85.10 \%)$ in the seven sentiments classified, outperforming shallow models (Naive Bayes and SVM) by approximately $34.53 \% \mathrm{p}$, and $7.14 \% \mathrm{p}$ respectively. These findings substantiate the applicability of the proposed Bi-LSTM-based sentiment classifier to the analysis of sentiments relevant to diverse climate change issues.

In the future, it will improve classification performance by improving deep neural network structure and parameters. Because CNN receives only text in one sentence, it is difficult to incorporate long-term dependency into the analysis. In the case of LSTM, it is difficult to capture sufficient lexical features because it relies entirely on embedding when text is entered. Therefore, to complement each model's weakness, we expect that the combined strengths of the individual models will improve performance. Also learning classification models on a consonant and vowel will minimize the pre-process and improve classification accuracy.

\section{ACKNOWLEDGMENT}

This study was conducted following the research work \ulcorner Big Data Analysis: Application to Environmental Research and Service (GP2018-13)」 and was funded by the Korea Environment Institute.

\section{REFERENCES}

1. K. H. Cheong, D. G. Lee, H. S. Ha, "Exploring Factors that Influence Trust in Local Government in South Korea"The Korean Association for Public Administration, 45(4), 2011, pp.181-202.

2. V. A. Chanley, "Trust in Government in the Aftermath of 9/11: Determinants and Consequences. Political psychology", 23(3), 2002, pp. 469-483.

3. T. M. Song, J Song, J. Y. An, L. L. Hayman, J. M.Woo, "Psychological and social factors affecting Internet searches on suicide in Korea: a big data analysis of Google search trends", Yonsei medical journal,55(1), 2014, pp.254-263.

4. Y. Xiao, K. Cho,"Efficient character-level document classification by combining convolution and recurrent layers", arXiv preprint arXiv:1602.00367, 2016

5. M. Cliché,"BB_twtr at SemEval-2017 Task 4: Twitter Sentiment Analysis with CNNs and LSTMs"arXiv preprint arXiv:1704.06125, 2017.

6. K. Kowsari, D. E. Brown, M. Heidarysafa, K. J. Meimandi, M. S. Gerber, L. E. Barnes, "Hdltex: Hierarchical deep learning for text classification" In Machine Learning and Applications (ICMLA), 2017 16th IEEE International Conference. 2017, pp. 364-371.

7. X. Zhang, J. Zhao,Y. LeCun, “Character-level convolutional networks for text classification". In Advances in neural information processing systems. 2015, pp.649-657.

8. B. Pang, L. Lee, S. Vaithyanathan, "Thumbs up?: sentiment classification using machine learning techniques", In Proceedings of the ACL-02 conference on Empirical methods in natural language processing-Volume 10. Association for Computational Linguistics. , 2002, pp.79-86.

9. Y. Wang, M. Huang, L. Zhao,"Attention-based lstm for aspect-level sentiment classification", In Proceedings of the 2016 Conference on Empirical Methods in Natural Language Processing.2016, pp. 606-615.

10. P. M. Sosa,"Twitter Sentiment Analysis using combined LSTM-CNN Models", academia.edu, 2017.

\section{AUTHORS PROFILE}

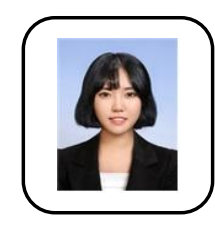

Do-Yeon Kim received Master's Degree of Business Administration from Chungbuk National University, South Korea. She is currently Researcher at Korea Environment Institute (KEI). Her present research interest include: Big Data, Machine Learning, Text Mining, Artificial Emotion Intelligence, Technology Innovation, MIS

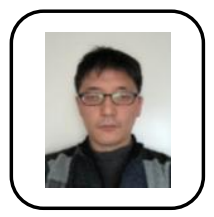

Sung-Won Kangreceived Ph.D. degrees from Rutgers, the State University of New Jersey, New Jersey, U.S.A. He is currently a senior researcher at Korea Environment Institute.His current research includes computable general equilibrium model, greenhouse gas emission regulation, and machine learning.

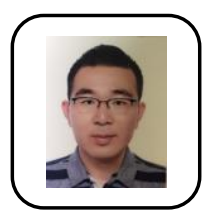

Seong-Taek Park received Ph.D. Degree in Management Information Systems from Chungbuk National Univeristy, South Korea. He is currently a Professor at The Korea Association of Software Manpower. His present research interests include: Big Data, Cloud Computing, Technology Innovation, MIS, Approriability Mechanism, Patent Valuation. 